\title{
Modic type I changes of the lumbar spine in golfers
}

\author{
Jason Mefford • Koichi Sairyo • Toshinori Sakai • \\ Justin Hopkins • Madoka Inoue • Rui Amari • \\ Nitin N. Bhatia $\cdot$ Akira Dezawa $\cdot$ Natsuo Yasui
}

Received: 16 September 2010 /Revised: 25 October 2010 /Accepted: 27 October 2010 /Published online: 24 November 2010

(C) The Author(s) 2010. This article is published with open access at Springerlink.com

\begin{abstract}
Low back pain (LBP) is the most prevalent musculoskeletal complaint among professional and amateur golfers; however, associated radiological changes in golfrelated LBP have not been examined in the literature. We suspect that Modic Type 1 changes in the lumbar spine are linked to golf-related LBP. In this retrospective case series, four middle-aged golfers (one professional and three high-level amateurs) presented to our clinic with LBP. Inflammation of the right side of endplates in the lumbar spine was suspected based on Modic Type 1 changes detected by magnetic resonance imaging (MRI) in each patient. All four cases were diagnosed with right-sided endplate inflammation and administered intradiscal steroid injections with a non-steroidal antiinflammatory drug (NSAID). Treatment swiftly alleviated LBP and diminished Modic Type 1 changes on follow-up MRI 3-6 months later in all four patients. We suggest that
\end{abstract}

J. Mefford · J. Hopkins

University of California,

Irvine, CA, USA

K. Sairyo $(\varangle) \cdot$ A. Dezawa

Department of Orthopaedic Surgery, School of Medicine,

University of Teikyo, Mizonokuchi Hospital,

Takatsu-ku, Kawasaki City, Japan

e-mail: sairyokun@hotmail.com

T. Sakai $\cdot$ M. Inoue $\cdot$ R. Amari $\cdot$ N. Yasui

Department of Orthopedics, Institute of Health Biosciences,

The University of Tokushima Graduate School,

Tokushima, Japan

T. Sakai $\cdot$ N. N. Bhatia

Department of Orthopedic Surgery, University of California,

Irvine, CA, USA
Modic Type 1 changes play a significant role in the diagnosis and treatment of golf-related LBP.

Keywords Low back pain - Golf - Modic Type 1 . Lumbar spine $\cdot$ MRI

\section{Introduction}

Golf is a widely popular sport boasting more than 27 million regular players in the U.S. [1]. Though physically less aggressive than other sports, golfers still sustain significant injuries [2]. The overwhelming majority of golfing injuries are attributed to the golf swing, usually from excessive use or poor swing mechanics $[3,4]$. Commonly injured areas include the wrist, elbow, and shoulder, although the predominant musculoskeletal complaint among professional and amateur golfers is low back pain (LBP) [4-7].

The golf swing imposes significant compression, anteriorposterior shearing, torsion, and lateral-bending forces on the lumbar spine [8], predisposing the lower back to a myriad of injures [9]. As the number of participants in golf continues to rise, sports medicine physicians should be familiar with common presentations of golf-related LBP and their radiological findings. Few reports however describe the radiological characteristics of LBP in golfers.

MRI is crucial to the standard evaluation of LBP [10]. More recently, vertebral endplate signal changes, or Modic changes [11], have been linked to LBP in occupational and clinical populations [12-18]. The relationship between Modic change and golf-related LBP, however, has yet to be reported. Here we present four cases of golf-related LBP in which Modic classification was employed as part of their evaluation. 


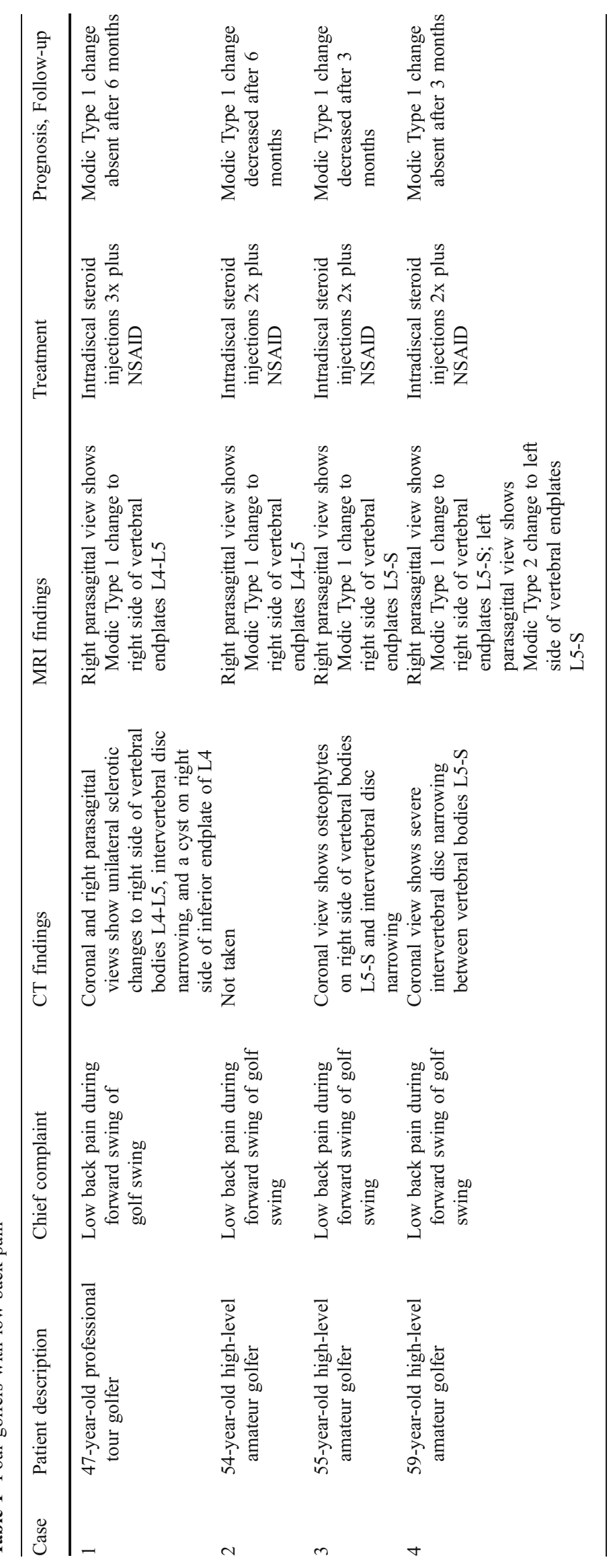




\section{Materials and methods}

We retrospectively investigated four middle-aged male golfers (one professional and three high-level amateurs) complaining of LBP (Table 1). The mean age of the patients was 53.8 years, ranging from 47 to 59 years. No patients had a history of scoliosis, limb length discrepancy, or hip pathology. To our knowledge they did not play other sports or experience any other injuries. Using T1 and T2weighted MRI findings plus short inversion-time inversion recovery (STIR) and fat saturation sequences, endplate changes in the lower spine were classified as Modic Type 1 (inflammation), Type 2 (fatty replacement), or Type 3 (sclerotic change) [11, 19]. Reconstruction computed tomography (CT) was utilized for further characterization of abnormalities.

\section{Results}

Case 1

A 47-year-old male professional tour golfer presented with a 6-year history of LBP. The primary aggravating factor was the forward swing of his golf swing, limiting his play to only nine holes of golf on an 18-hole course [20-22]. Apart from golf, the patient did not experience any LBP and was otherwise in good general health.
Fig. 1 A Right parasagittal MRI shows a Modic Type 1 change $(a, b)$. Short time inversion recovery (STIR) showing high vertebral endplate signal intensity at the L4-L5 level (c). B Coronal and right parasagittal CT findings show unilateral and sclerotic endplate changes at the L4-L5 level, intervertebral disc narrowing, and a cyst located on the right inferior endplate of vertebral body L4. C Right parasagittal STIR MRI 6 months after initial presentation shows disappearance of original vertebral endplate signal hyper-intensity at the L4-L5 level

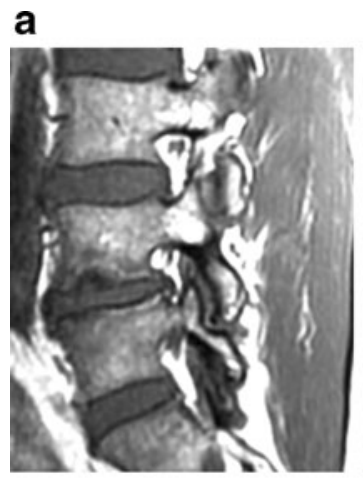

a
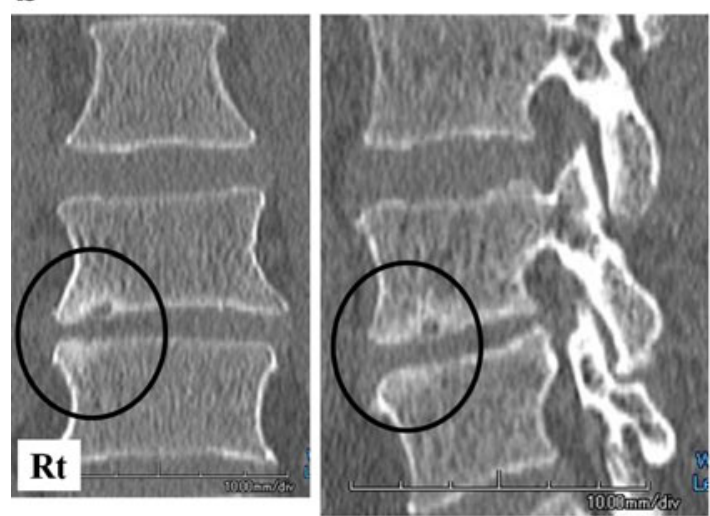

b

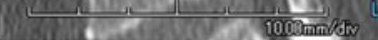

c

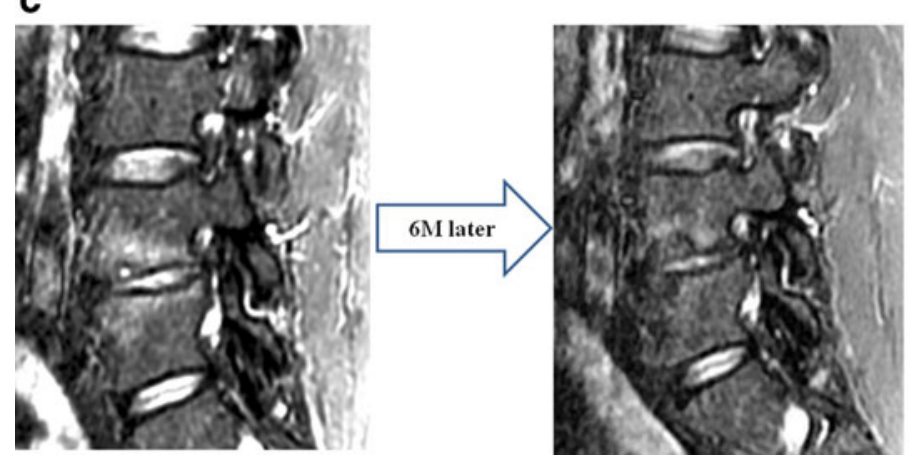

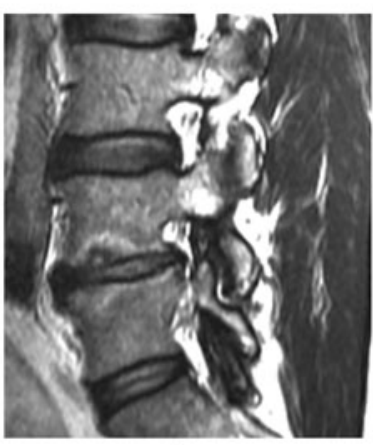

b

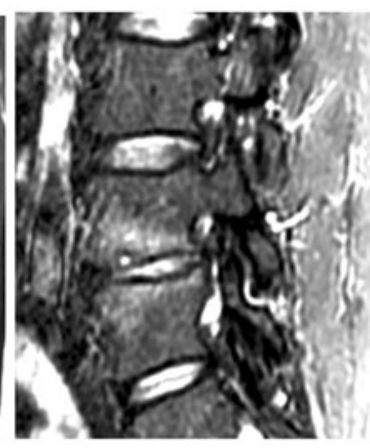

c 

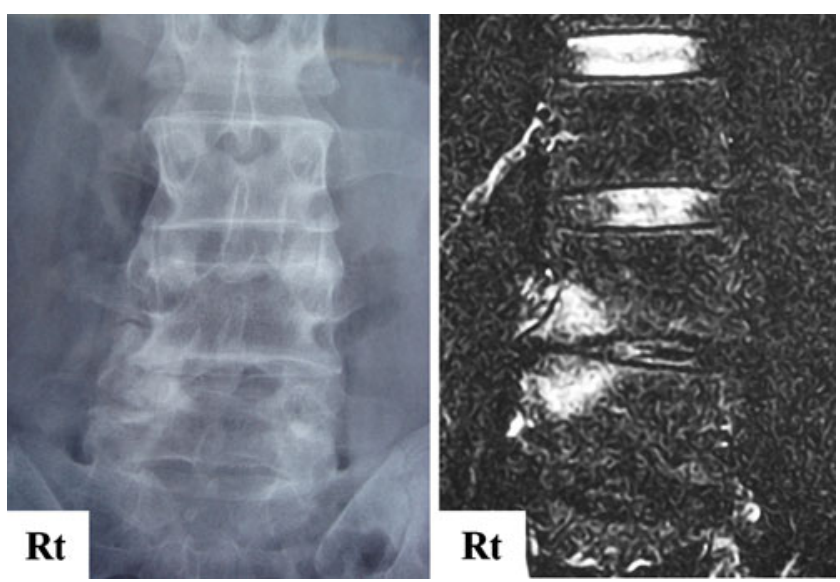

Fig. 2 AP radiograph shows unilateral and sclerotic endplate changes at the L4-L5 level and intervertebral disc narrowing. Coronal T2weighted image with fat suppression shows high vertebral endplate signal intensity at the L4-L5 level
On physical examination, straight leg raise test was negative on the left and right to $90^{\circ}$. Femoral nerve stretch test and neurological examination were negative. Sensory function was intact and all reflexes were found to be normoactive.

Analysis of right parasagittal T1 and T2-weighted MRI with STIR revealed unilateral Modic Type 1 changes at the L4L5 level in the patient's lumbar spine (Fig. 1A). Coronal and right parasagittal $\mathrm{CT}$ findings showed unilateral and sclerotic endplate changes at the L4-L5 level, intervertebral disc narrowing, and a cyst located on the right inferior endplate of vertebral body L4 (Fig. 1B). The patient was diagnosed with right-sided endplate inflammation and administered three intradiscal steroid injections to the affected endplates at 1-month intervals. An NSAID was prescribed in conjunction with the treatment. Shortly after the first intradiscal steroid injection, complete alleviation of his LBP was obtained. A 6-month follow-up MRI confirmed the disappearance of the original Modic Type 1 changes (Fig. 1C). Currently, he was able to resume playing all 18 holes of golf.
Fig. 3 A Coronal CT scan shows osteophytes on the right side of vertebral endplates L4 and L5, as well as intervertebral disc narrowing. B Right parasagittal MRI shows a Modic Type 1 change. STIR MRI shows high vertebral endplate signal intensity on the right side at the L5-S level. C A 3-month follow-up MRI confirmed the reduction of Modic Type 1 changes

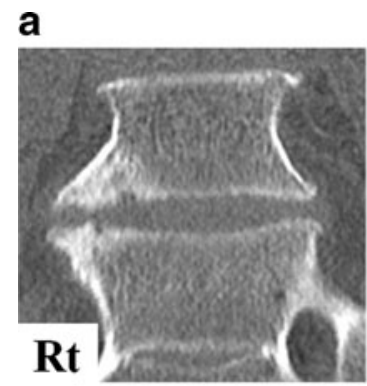

b

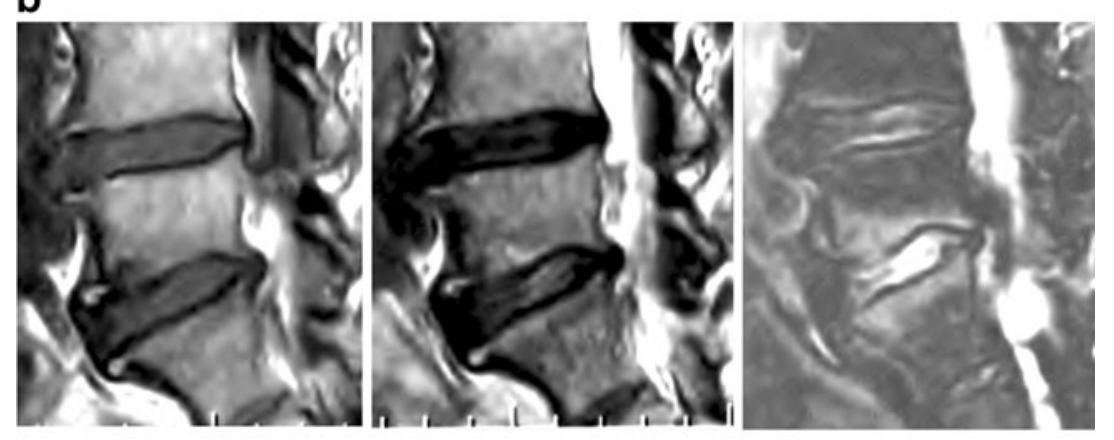

c

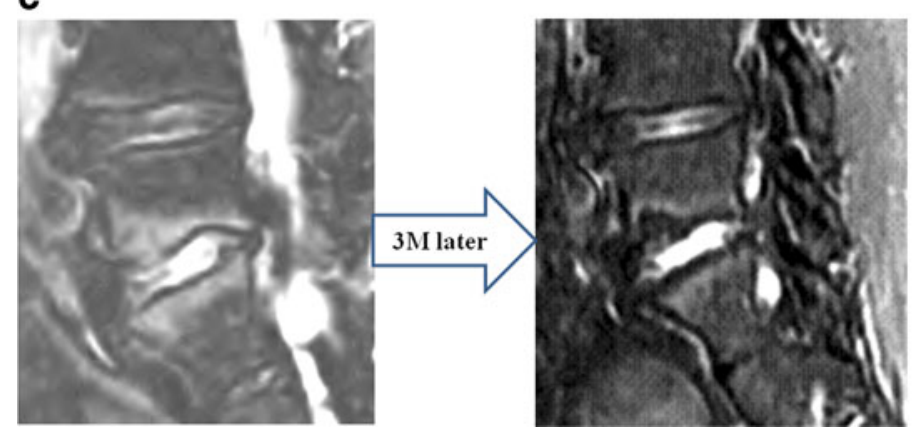




\section{Case 2}

A 54-year-old male high-level amateur golfer presented with a several-year history of LBP and the same aggravating factors as described in Case 1. AP radiograph showed unilateral intervertebral disc narrowing and sclerotic changes at the L4-L5 level, which corresponded to high vertebral endplate signal intensity on a coronal T2-weighted image with fat suppression (Fig. 2).

Diagnosis and treatment were the same as in Case 1. Shortly after the first intradiscal steroid injection, complete alleviation of his LBP was obtained.

Case 3

A 55-year-old male high-level amateur golfer presented with a several-year history of LBP and the same aggravating factors described in Case 1. Coronal CT scan showed osteophytes on the right side of vertebral endplates at the L5-S level, as well as intervertebral disc narrowing (Fig. 3A). MRI revealed Modic Type 1 changes that mimicked the first two cases as well (Fig. 3B). Diagnosis and treatment were the same as in Case 1. Shortly after the first intradiscal steroid injection, complete alleviation of his LBP was obtained. A 3-month follow-up MRI confirmed the reduction of Modic Type 1 changes (Fig. 3C).

Case 4

A 59-year-old male high-level amateur golfer presented with a several-year history of LBP and the same aggravating factors as described in Case 1. Coronal CT findings revealed severe intervertebral disc narrowing at the L5-S1 level (Fig. 4A). MRI revealed unilateral and multilevel (L5S1) Modic Type 1 changes to the right side of the patient's lumbar spine (Fig. 4B). Additionally, Modic Type 2 endplate changes were observed on the left side of the same vertebrae. Diagnosis and treatment were the same as in Case 1. Shortly after the first intradiscal steroid injection, complete alleviation of his LBP was obtained.

These patients and their families were informed that data from their cases would be submitted for publication and gave their consent.
Fig. 4 A Coronal CT findings revealed severe intervertebral disc narrowing at L5-S1. B MRI revealed unilateral and multilevel (L5-S1) Modic Type 1 changes to the right side of the patient's lumbar spine, while Modic Type 2 endplate changes were observed on the left side of the same vertebrae

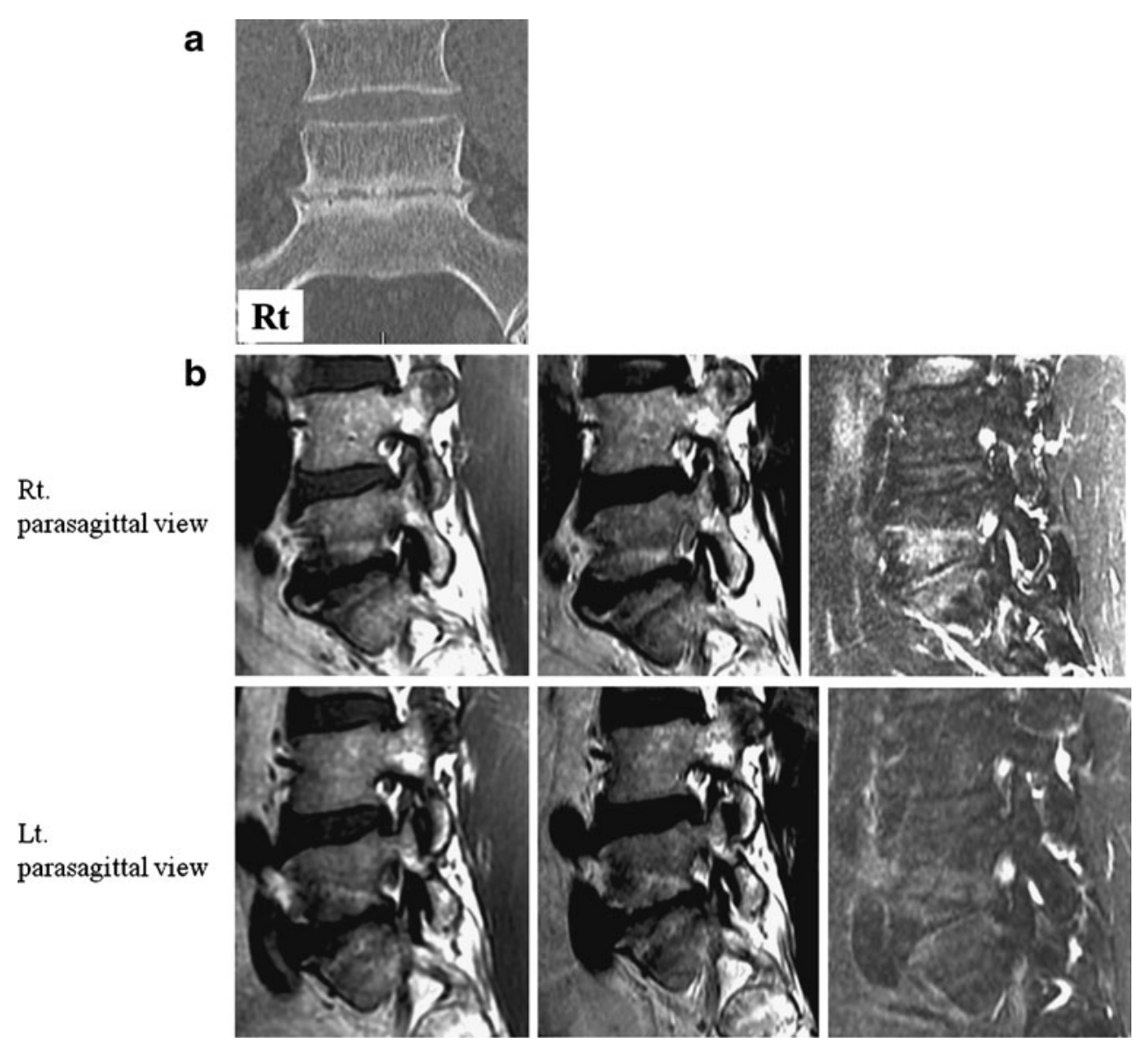




\section{Discussion}

The golf swing is responsible for the majority of golfrelated injuries usually from overuse or poor swing mechanics $[3,4]$. According to the literature, the swing can be broken down into four basic stages: the backswing or take away, the forward swing, the acceleration or impact phase, and the early and late follow-through [3, 20-23]. All patients in this study experienced LBP during the forward swing. Pink et al. analyzed the muscle-firing patterns of 23 golfers with handicaps of five or below. In their analysis, the erector muscles of the right side peaked during the forward swing [21]. This repetitive movement could cause the right-sided trunk injury and endplate inflammation found in the present cases.

LBP in golfers can originate from several types of swing-related injuries including muscle strains, facet arthropathy, herniated nucleus pulposus, spondylolisthesis, and stress fractures of the vertebral body and pars interarticularis [9]. All patients in this study were diagnosed with endplate inflammation of the lumbar spine specifically localized to the right side. An anterior view threedimensional finite element lumbar spine model at L3 to S1 showed increased stress around the right side of vertebral endplates during right bending (Fig. 5). This coincided with inflammation detected in the same area on MRI suggesting a biomechanical relationship between the forward swing and LBP. Most golfers, including our

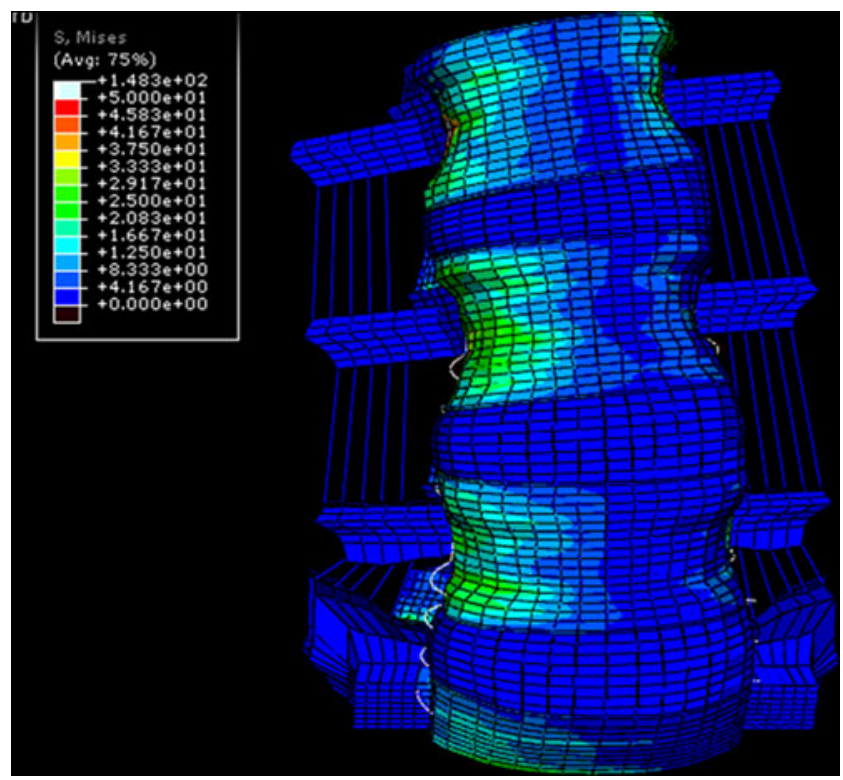

Fig. 5 Finite element lumbar spine model at L3 to S1 shows increased stress on the right side around vertebral endplates during right lateral bending patients and other professionals, use a right-handed stance to swing the golf club [20]. Assuming the injury is linked to the biomechanics of right-handedness, one would predict right-sided endplate inflammation to be a relatively common cause of golf-related LBP. However, reports of this injury were not found in our review of the literature. It is believed that inflammation, characterized by Modic Type 1 changes on MRI, arises on the right side of vertebral endplates of lumbar vertebrae as the result of repetitive compression, shearing and torsion forces placed there during the forward swing of a righthanded golfer. Nonetheless, further investigation into the etiology of right-sided endplate inflammation, as it pertains to the biomechanics of a right-handed golf swing, is warranted.

MRI is ideal for detecting disc and subchondral bone marrow signal changes [24, 25]. Modic changes as first described by Modic et al. are characterized by comparing vertebral endplate signal changes on $\mathrm{T} 1$ and $\mathrm{T} 2$-weighted MRI. These comparisons are then categorized into one of three types: Type 1 (low intensity, high intensity), Type 2 (high intensity, high intensity), or Type 3 (low intensity, low intensity) [11, 19]. Modic Type 1 changes are generally associated with inflammation or neovascularity mediated by pro-inflammatory cytokines $[11,19,26]$. This would account for LBP in the present cases Moreover, asymptomatic patients exhibiting Modic Type 1 changes are very rare [27]. In this study, successful treatment of affected endplates was guided by Modic Type 1 changes, suggesting that Modic classification has potential to be an effective diagnostic tool for LBP in golfers. Appreciating that lower back injuries account for 15-34\% of all injuries in amateurs and $22-24 \%$ of all injuries in professionals $[3,4,6,7,28$, 29], the potential of Modic change as a diagnostic tool in golf-related LBP demands further study.

\section{Conclusions}

Four cases of golf-related LBP exhibiting Modic Type 1 changes in the lumbar spine were diagnosed with rightsided endplate inflammation. Treatment with intradiscal steroid injections swiftly alleviated LBP and led to a disappearance of Modic Type 1 changes on follow-up MRI in all four patients. Therefore, we suggest that Modic Type 1 changes are considered in the diagnosis and treatment of LBP in golfers. Continued biomechanical research into the golf swing's different stages and association to right-sided endplate inflammation will provide further knowledge necessary to determine the exact role of Modic change in golf-related LBP. 
Open Access This article is distributed under the terms of the Creative Commons Attribution Noncommercial License which permits any noncommercial use, distribution, and reproduction in any medium, provided the original author(s) and source are credited.

\section{References}

1. National Golf Foundation Questions/FAQs. Available at: http:// www.ngf.org/cgi/whofaqa.asp. 2010; Accessed July 29.

2. McCarroll JR. The frequency of golf injuries. Clin Sports Med. 1996;15(1):1-7.

3. McCarroll JR. Overuse injuries of the upper extremity in golf. Clin Sports Med. 2001;20(3):469-79.

4. McCarroll JR, Gioe TJ. Professional golfers and the price they pay. Physician Sportsmed. 1982;10:64-70.

5. Batt ME. A survey of golf injuries in amateur golfers. Br J Sports Med. 1992;26:63-5.

6. Batt ME. Golfing injuries. An overview. Sports Med. 1993;16 (1):64-71.

7. McCarroll JR, Rettig AC, Shelbourne KD. Injuries in the amateur golfer. Phys Sportsmed. 1990;18(3):122-6.

8. Stover CN MJ, Mallon WJ. Feeling up to par: medicine from tee to green. Philadelphia: FA Davis; 1994.

9. Hosea TM, Gatt CJ. Back pain in golf. Clin Sports Med. 1996;15 (1):37-53

10. Adams MA, Freeman BJC, Morrison HP, et al. Mechanical initiation of intervertebral disc degeneration. Spine (Phila $\mathrm{Pa}$ 1976). 2000;25(13):1625-36.

11. Modic MT, Steinberg PM, Ross JS, et al. Degenerative disk disease - Assessment of changes in vertebral body marrow with MR imaging. Radiology. 1988;166(1):193-9.

12. Albert $\mathrm{H}$, Manniche C. Modic changes following lumbar disc herniation. Eur Spine. 2007;16(7):977-82.

13. Braithwaite I, White J, Saifuddin A, et al. Vertebral end-plate (Modic) changes on lumbar spine MRI: correlation with pain reproduction at lumbar discography. Eur Spine. 1998;7(5):363-8.

14. Cvitanic OA, Schimandle J, Casper GD, et al. Subchondral marrow changes after laser diskectomy in the lumbar spine: MR Imaging findings and clinical correlation. AJR Am J Roentgenol. 2000;174(5):1363-9.

15. Ito M, Incorvaia KM, Yu SF, et al. Predictive signs of discogenic lumbar pain on magnetic resonance imaging with discography correlation. Spine (Phila Pa 1976). 1998;23(11):1252-8.
16. Kuisma M, Karppinen J, Niinimäki J, et al. Modic changes in endplates of lumbar vertebral bodies: prevalence and association with low back and sciatic pain among middle-aged male workers. Spine (Phila Pa 1976). 2007;32(10):1116-22.

17. Schenk P, Läubli T, Hodler J, et al. Magnetic resonance imaging of the lumbar spine: findings in female subjects from administrative and nursing professions. Spine (Phila Pa 1976). 2006;31 (23):2701-6.

18. Weishaupt D, Zanetti M, Hodler J, et al. Painful lumbar disk derangement: relevance of endplate abnormalities at MR imaging. Radiology. 2001;218(2):420-7.

19. Modic MT, Masaryk TJ, Ross JS, et al. Imaging of degenerative disk disease. Radiology. 1988;168(1):177-86.

20. Adlington GS. Proper swing technique and biomechanics of golf. Clin Sports Med. 1996;15(1):9-26.

21. Pink M, Perry J, Jobe FW. Electromyographic analysis of the trunk in golfers. Am J Sports Med. 1993;21(3):385-8.

22. Watkins RG, Uppal GS, Perry J, et al. Dynamic electromyographic analysis of trunk musculature in professional golfers. Am J Sports Med. 1996;24(4):535-8.

23. Theriault G, Lachance P. Golf injuries - An overview. Sports Med. 1998;26(1):43-57.

24. Jinkins JR, Van Goethem JWM. The postsurgical lumbosacral spine - Magnetic resonance imaging evaluation following intervertebral disk surgery, surgical decompression, intervertebral bony fusion, and spinal instrumentation. Radiol Clin North Am. 2001;39(1):1-29.

25. Tehranzadeh J, Andrews C, Wong E. Lumbar spine imaging. Normal variants, imaging pitfalls, and artifacts. Radiol Clin North Am. 2000;38(6):1207-53.

26. Ohtori $\mathrm{S}$, Inoue $\mathrm{G}$, Ito $\mathrm{T}$, et al. Tumor necrosis factorimmunoreactive cells and PGP 9.5-immunoreactive nerve fibers in vertebral endplates of patients with discogenic low back pain and Modic Type 1 or Type 2 changes on MRI. Spine (Phila Pa 1976). 2006;31(9):1026-31.

27. Weishaupt D, Zanetti M, Hodler J, et al. MR imaging of the lumbar spine: Prevalence of intervertebral disk extrusion and sequestration, nerve root compression, end plate abnormalities, and osteoarthritis of the facet joints in asymptomatic volunteers. Radiology. 1998;209(3):661-6.

28. Gosheger G, Liem D, Ludwig K, et al. Injuries and overuse syndromes in golf. Am J Sports Med. 2003;31(3):43843.

29. McHardy A, Pollard H, Lu K. Golf injuries: A review of the literature. Sports Med. 2006;36(2):171-87. 